\author{
Allison C.E. Bidulock ${ }^{1,2}$ \\ Pavel Dubský2 (iD \\ Albert van den Berg ${ }^{1}$ \\ Jan C.T. Eijkel ${ }^{1}$ \\ ${ }^{1}$ BIOS-Lab on a Chip Group, \\ MESA+ Institute of \\ Nanotechnology, TechMed \\ Centre and Max Planck Center \\ for Complex Fluid Dynamics, \\ University of Twente, Enschede, \\ Overijssel, The Netherlands \\ ${ }^{2}$ Department of Physical and \\ Macromolecular Chemistry, \\ Charles University in Prague, \\ Prague, Czech Republic
}

Received September 17, 2018

Revised December 7, 2018

Accepted December 7, 2018

\section{Research Article \\ Integrated internal standards: A sample prep-free method for better precision in microchip CE}

Point-of-care systems based on microchip capillary electrophoresis require single-use, disposable microchips prefilled with all necessary solutions so an untrained operator only needs to apply the sample and perform the analysis. While microchip fabrication can be (and has been) standardized, some manufacturing differences between microchips are unavoidable. To improve analyte precision without increasing device costs or introducing additional error sources, we recently proposed the use of integrated internal standards (ISTDs): ions added to the BGE in small concentrations which form system peaks in the electropherogram that can be used as a measurement reference. Here, we further expand this initial proof-of-principle test to study a clinically-relevant application of $\mathrm{K}$ ion concentrations in human blood; however, using a mock blood solution instead of real samples to avoid interference from other obstacles (e.g. cell lysis). Cs as an integrated ISTD improves repeatability of $\mathrm{K}$ ion migration times from $6.97 \%$ to $0.89 \%$ and the linear calibration correlation coefficient $\left(R^{2}\right)$ for K quantification from 0.851 to 0.967 . Peak area repeatability improves from $11.6-13.3 \%$ to $4.75-5.04 \%$ at each $\mathrm{K}$ concentration above the LOQ. These results further validate the feasibility of using integrated ISTDs to improve imprecision in disposable microchip CE devices by demonstrating their application for physiological samples.

Keywords:

Microchip capillary electrophoresis / Precision / Quantification / Reproducibility DOI 10.1002/elps.201800393

Additional supporting information may be found online in the Supporting Information section at the end of the article.

\section{Introduction}

Point-of-care tests aim to perform a rapid measurement in near proximity to the patient (e.g. doctor's office, hospital room) or by the patient himself (e.g. at home), assisting in "bed-side" diagnosis and optimal treatment. Microchip Capillary Electrophoresis (microchip CE) lends itself well to pointof-care determination due to its modest instrumental needs: high voltage sources and conductivity detection circuitry can be easily downscaled. For developments in this area, including the application to point-of-care diagnostics, recent reviews can be consulted [1-4].

Correspondence: Dr. Pavel Dubský, Department of Physical and Macromolecular Chemistry, Charles University in Prague, Albertov 2030, Prague 128 43, Czech Republic

E-mail: dubsky@natur.cuni.cz

Abbreviations: His, L-histidine; HPMC, (hydroxypropyl)methyl cellulose; ISTD, internal standard; LTEM, linear theory of electromigration; PAR, peak area ratio
For a true point-of-care system based on microchip CE, the simplest implementation is one portable electronic system ("reader") per user that measures samples applied to multiple single-use, disposable microchips ("cartridges") prefilled with BGE. The company Medimate published several papers from 2010 to 2015 reporting on the performance of such a commercial system, initially based on the developments of Vrouwe et al. [5] in measuring Li+ ions in human serum (used in the treatment of manic depression), and then showing application to other analytes than $\mathrm{Li}+[6-8]$. While thermosets and thermoplastics are cost-effective alternatives for disposable microchip CE chips, glass still has the best performance [9]. Glass microchips are produced in the cleanroom by wet etching of fluidic channels, deposition of metal electrodes, powder blasting of reservoirs, and/or other openings, and high-temperature fusion bonding to close the device. Although cleanroom production procedures have been

Color online: See the article online to view Figs. 1-5 in color. 
standardized, some chip-to-chip variation is bound to remain, leading to decreased precision and accuracy.

To reduce residual error, it is common practice in CE [10-12] and microchip CE $[13,14]$ to add an internal standard (ISTD) to the sample. In a recent paper, we verified the significance of chip-to-chip differences in quantification imprecision with a systematic investigation across six microfluidic chips, and further determined how well two ISTDs (Cs and Li) accounted for this error [15]. Unfortunately, the addition of an ISTD to the sample still poses a major problem for point-of-care analysis. If the ISTD were to be added to the sample outside the microchip, it would further complicate the measurement and make repeatable analysis by an unskilled user impossible. If the ISTD were to be stored in the glass chip together with the prefilled BGE for simple operator handling, a reliable mixing/sample preparation step would be needed, introducing additional error sources and requiring more space on the already expensive glass device. To avoid these complications and still add an ISTD, we recently proposed an alternative method whereby the ISTD is added to the BGE instead of to the sample [16]. The added ion in the BGE results in a new system peak in the electropherogram [17], which we proposed could then be used as a reference or "integrated ISTD" peak. Such system peaks consist of BGE ions like the rest of the separation channel but in different concentrations formed by the discontinuity of the sample plug in the separation channel. In this paper, we will denote the system peak we use as an integrated ISTD peak with quotations around the BGE-added ion; for example, as a "Cs" peak when $\mathrm{Cs}+$ ions are added to the BGE.

In the previous paper [16], we found for a proof-ofprinciple sample that the integrated ISTD peaks moved with similar mobility and peak shape to the sample-added traditional ISTDs. Both "Cs" and "Li" were found to be well correlated to the $\mathrm{Na}$ analyte (in contrast to the conventional use of ISTDs where Cs was better correlated than Li), suggesting the method might be non-selective. Final corrected RSD values were as good or better than the conventional ISTD addition method; however, it should be noted that the uncorrected Na analyte RSDs also improved, seemingly due to reduced surface-analyte interactions. One concern that arose from this simple study was that the integrated ISTD peak magnitudes were strongly dependent on both the BGE and sample matrix, rather than being independent of the sample matrix as with a conventional ISTD. As the composition of the sample changed (by varying the concentration of the analyte), the heights and areas of the integrated ISTD peaks changed as well, resulting in nonlinear calibration curves for the peak ratio correction. While we demonstrated this did not eliminate the possibility of using the BGE-added standards as a correction factor for the measurement, the question naturally arises how the method would perform with a more clinically relevant sample of less variable composition; for example, human blood.

Ionic concentrations in human blood are strictly controlled within a few mmol/L [18]: $\mathrm{Na}+$ between 135$145 \mathrm{mmol} / \mathrm{L} ; \mathrm{K}+$, 3.5-5.0 mmol/L; Ca2+, 2.2-2.65 mmol/L;
Mg2+, 0.6-1.1 mmol/L. With the large amount of $\mathrm{Na}+\mathrm{com}$ pared to other ions, even with major fluctuations in the other ions $(\mathrm{K}+, \mathrm{Ca} 2+, \mathrm{Mg} 2+)$, the size of the BGE-added integrated ISTD should vary much less. Thus, the behavior of the integrated ISTD would be expected to perform closer to that of a sample-added ISTD under these conditions, resulting in linear calibration curves. To investigate this, we explore the effect of the "Cs" integrated ISTD peak on the chip-tochip reproducibility of $\mathrm{K}+$ ion quantification, using a solution containing cation concentrations similar to the normal ranges in human blood plasma. As in previous work $[15,16]$, we aimed to systematically measure six concentrations of $\mathrm{K}+$ ions, 30 times per each concentration (six sample loads of five injection-separation pairs each) on each microchip, of which there were also six. This resulted in 1080 electropherograms from which we can draw reliable conclusions about the system's precision for $\mathrm{K}+$ ion quantification-and more importantly-how that precision is corrected with the integrated ISTD.

This investigation has a direct clinical relevance. Hyperkalemia is a medical condition where too many $\mathrm{K}+$ ions are present in the blood and is seen frequently in hospital Emergency departments. Typically diagnosed from blood serum samples, moderate hyperkalemia is defined as a $\mathrm{K}+$ ion concentration of above $6 \mathrm{mmol} / \mathrm{L}$, with severe hyperkalemia defined as above $7 \mathrm{mmol} / \mathrm{L}$. Outward symptoms are typically not present until the hyperkalemia is severe, at which point the cardiovascular system can become compromised, subsequently leading to death. Thus, a device that can quickly diagnose this condition at the patient's bedside in the Emergency room would be beneficial. We acknowledge many obstacles exist in the development of such a point-of-care system (such as cell lysis) that are not addressed in this paper. Its aim is rather to investigate the use of a BGE-integrated ISTD to improve the quantification precision, which is a very likely hurdle for bedside measurements with single-use microchips.

\section{Materials and methods}

\subsection{Microchip CE system}

The same setup, chip holders, and experimental procedure was used here as in previous work $[15,16]$, with some exceptions.

The high voltage system was replaced with an instrument capable of operating at higher voltages and collecting the current information (HVS448 3000-LC, LabSmith Inc., Livermore CA, USA), which allowed us to increase the voltage to perform faster separation steps. The pinched injection protocol remained the same as in previous work: $1000 \mathrm{~V}$ applied to the sample inlet and BGE outlet, $800 \mathrm{~V}$ to the BGE inlet, and $0 \mathrm{~V}$ to the sample waste reservoir. Separation then applied $1500 \mathrm{~V}$ to the BGE inlet, $975 \mathrm{~V}$ to the sample inlet, $950 \mathrm{~V}$ to the waste reservoir, and $0 \mathrm{~V}$ to the BGE outlet. Higher separation voltages would sometimes result in the formation of a gas bubble between the thin-film conductivity electrodes 
due to the electrolysis of water, invalidating the measurement. Injection and separation times were 45 and $35 \mathrm{~s}$, respectively, for all electrophoretic runs. It should be noted the protocol was not optimized for measurement repeatability.

Six Borofloat ${ }^{\circledR}$ glass CE microchips were chosen from the same initial batches (wafer sets) fabricated in-house for the original work [15], of which only one chip was used in previous experiments (3-2). Channels were $6 \mu \mathrm{m}$ deep by $52 \mu \mathrm{m}$ wide; $140 \mathrm{~nm}$ recessed platinum electrodes were in contact with the solution, $2 \mathrm{~cm}$ from the double-T intersection; each chip had dimensions of $15 \times 30 \times 1.6 \mathrm{~mm}$. Microchips were cleaned by flowing $0.1 \mathrm{~N} \mathrm{NaOH}$ and water through the channels as in previous work, and then were left to acclimatize to the BGE buffer constituents (sans reference standard) for at least 90 minutes. Microchips were then filled with, and stored in, Milli-Q water.

For further details on the microchip fabrication, chip holders, and detection electronics, please see previous work [15].

\subsection{Reagents}

BGE solutions were mixed daily from stock solutions and consisted of $120 \mathrm{mM}$ MES-His (L-histidine), 0.01\% w/v (hydroxypropyl)methylcellulose (HPMC), $2.5 \mathrm{mM}$ 18-crown-6 (to effectively resolve the $\mathrm{K}$ peak from the "Cs" peak), $5 \mathrm{mM}$ of $\mathrm{NaCl}$ (to improve the analyte peak shape), and $1 \mathrm{mM}$ of the $\mathrm{CsCl}$ reference standard. The $250 \mathrm{mM}$ MES-His stock solution was verified to have a $\mathrm{pH}$ of $\sim 6.1$ at room temperature after mixing. Details on the design choices for this BGE solution are given in Results and Discussion. Sample solutions were also prepared from stock daily and contained $140 \mathrm{mM} \mathrm{NaCl}, 0.01 \% \mathrm{HPMC}, 2 \mathrm{mM} \mathrm{CaCl}_{2}, 1 \mathrm{mM} \mathrm{MgCl}_{2}$, and one of six different concentrations of $\mathrm{KCl}(3,4,5,6,7$, and $8 \mathrm{mM}$ ). Chemicals were all $99 \%$ grade or higher and, except for the LiCl stock solution donated by Medimate (Enschede, The Netherlands), were purchased from Sigma-Aldrich (Steinheim, Germany). Solutions were mixed with Milli-Q water.

\subsection{Experimental procedure}

A "sample load" is defined as one application of sample solution to the microchip, which five injection-separation pairs or "runs" are then performed on six sample loads were performed for each of six concentrations of $\mathrm{KCl}$ on each of six microchips: totaling of 1080 electropherograms.

Immediately prior to the sample load, all chip reservoirs were washed $3 \mathrm{x}$ with Milli-Q water and then the reservoir at the end of the separation channel (buffer waste) was washed $3 \mathrm{x}$ with BGE. This reservoir was then filled will BGE and negative pressure was applied to the other three reservoirs for 10 minutes, replenishing BGE in the microchannels. These three reservoirs were then washed $3 \mathrm{x}$ with BGE and the filled reservoir was emptied. The $55 \mu \mathrm{L}$ of sample and BGE were then immediately applied to their respective wells.

\subsection{Baseline fit and peak determination}

As in previous papers, all electropherograms were batch processed using a single MATLAB script, rather than manually determined. When considering the application-disposable microfluidic chips for point-of-care measurements-trainedoperator peak determination is not an available option.

Unfortunately, the very broad and slow-moving Na peak made it difficult to reliably fit the signal's baseline with the background correction method developed by V. Mazet et al. (in literature [19] and also available on MATLAB's File Exchange) without additional processing. Thus, the following algorithm was designed. After simple Butterworth signal filtering, the electropherogram data was truncated from $\sim 2 \mathrm{~s}$ after the high voltages were switched to the beginning of the Na peak. A symmetrical Huber function with a polynomial order of 2 and a threshold of 0.001 was first used to approximate the baseline. While it matched the start of the electropherogram well, the fit often over- or undershot the end of the truncated signal (the baseline between $\mathrm{K}$ and $\mathrm{Na}$ peaks). To correct this, the region from the "Cs" peak's leading edge to the truncated signal's end was multiplied by an incremental scalar array. In essence, it was designed to push the fit down/up to the end of the signal, using the baseline immediately before the "Cs" as a pivot point. This procedure is illustrated in Fig. 1.

Peak heights were taken from the peak's maximum point to zero after background correction. Peak areas were determined using MATLAB's trapezoidal numerical integration function. The start point of the peak was chosen as the

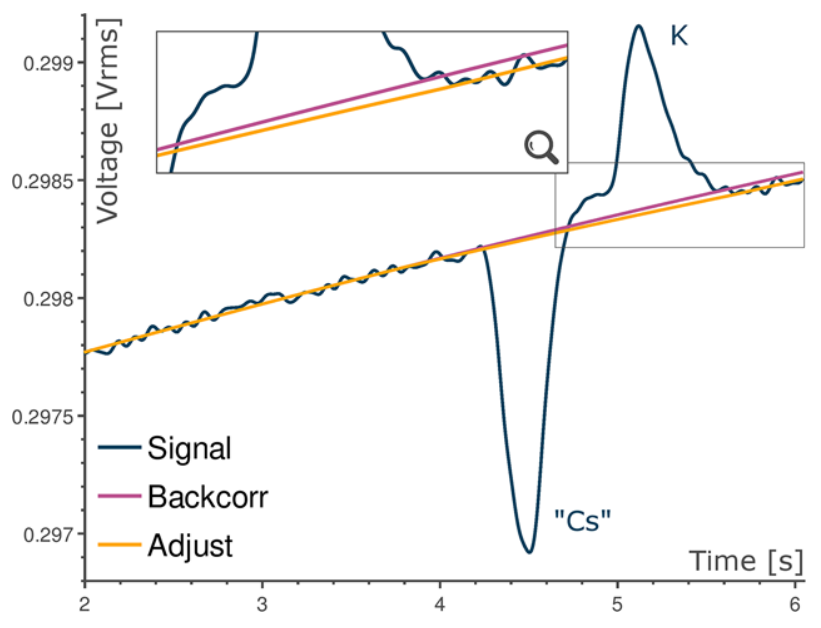

Figure 1. Two baseline fits to the same electropherogram: "Backcorr" using the background correction method from MATLAB's File Exchange; "Adjust" using the average signal baseline magnitude after the $K$ peak to bend the fit down, using the "Cs" start as a pivot point. This better approximates the baseline, leading to improved RSD values. 
zero-crossing point of the corrected signal's derivative on the leading edge; the end point was chosen as the zero-crossing point of the baseline subtracted signal itself on the tailing edge. Peaks often overshoot the baseline on the tailing edge before easing back into it, suggesting capacitive effects in the detection system. The end point was chosen in this way to ignore these effects. Additional area calculation methods were investigated, including the full width at half maximum estimation used in previous work; however, this method proved less reproducible here, likely due to the asymmetrical $K$ peaks.

\subsection{Simul 5}

All simulations performed in this paper are done using the freeware program Simul 5 [20]. Using mathematical models, the software simulates a spatial image of the electromigration and diffusion of electrolytes in free solution, while preserving mass conservation, acid-base equilibria, and electroneutrality laws. It provides electrophoresis researchers with a platform to inspect system peaks, sample (de)stacking, and helps optimize experimental conditions.

With its intersecting channels design, the microchip architecture poses a $2 \mathrm{D}$ simulation problem that cannot be directly handled by Simul 5 since it only models a single capillary. However, since one channel is used for electrokinetically injecting the sample, while the second channel is used for separating the injected sample plug (where the sample plug width is determined by the cross section of the two channels), it is possible to use a two-step simulation to model each channel individually using Simul 5. First, the injection step was modeled by breaking the capillary into two segments, with the sample in one segment and the BGE in the other. The sample was then electrokinetically injected into the BGE zone, and the program was left to run until all migrating constituents had crossed the stationary boundary and traveled well into the BGE segment (empirically visible as a very distinct plateau region extending from the stationary boundary to the beginning of the slowest migrating ion). Electrokinetic injection mobility bias is avoided in microchip CE by ensuring the injection time is sufficiently long that all migrating species have had time to cross the channel intersection, while preventing diffusion into the separation channel. Thus, the composition of this simulated plateau region can be used to model the composition of the sample plug in the separation step, which is then performed as a typical 1-dimensional Simul 5 simulation.

In the simulations performed here, the effects of ionic strength were not accounted for. This is due to the heavy computing stress these calculations require and negative value errors resulting from spurious oscillations formed at sharp boundaries. Thus, the Ca and $\mathrm{Mg}$ peaks should be disregarded in all simulated electropherograms. These peaks have slower migration times under these conditions due to their sensitivity to ionic strength, and thus appear later in the experimental electropherograms (hidden within the Na peak).

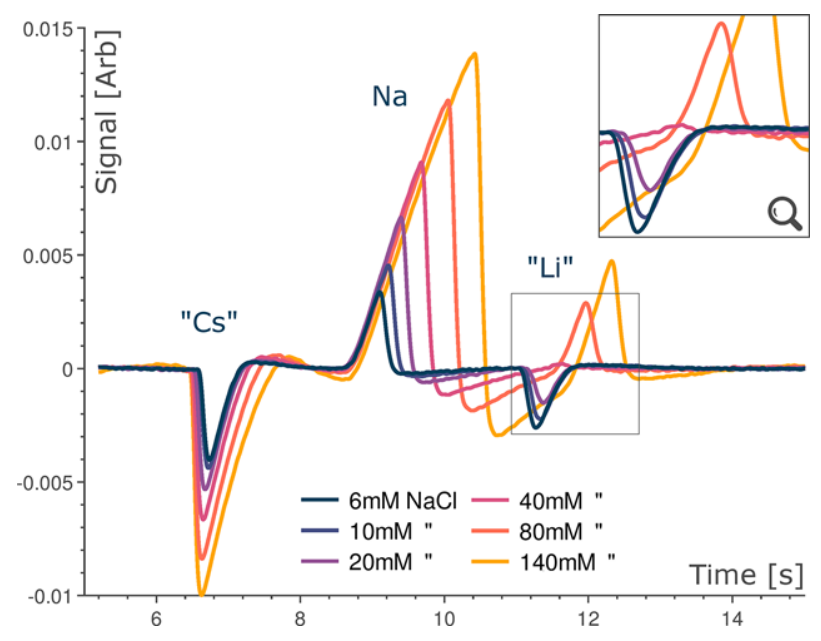

Figure 2. Experiments showing how the " $\mathrm{Cs}$ " and " $\mathrm{Li}$ " integrated ISTD peaks change in amplitude and area as the $\mathrm{Na}$ concentration increase in the sample. BGE: $100 \mathrm{mM}$ MES-His, 0.01\% HPMC, $0.25 \mathrm{mM} \mathrm{KCl}$, and $7.5 \mathrm{mM}$ of $\mathrm{CsCl}$ and $\mathrm{LiCl}$. Sample: $100 \mathrm{mM}$ MES-His, $0.01 \%$ HPMC, and varied $\mathrm{NaCl}$ concentration as shown on the figure's legend.

\section{Results and discussion}

\subsection{Preliminary experiments of increased $\mathrm{NaCl}$ sample concentration}

Starting with the BGE developed in previous work [16], the $\mathrm{NaCl}$ concentration was increased from 6 to $140 \mathrm{mM}$ to visualize how the integrated ISTD peaks would be influenced by high $\mathrm{Na}+$ ion concentrations as in blood. Figure 2 plots six electropherograms taken from the same chip with a BGE of $100 \mathrm{mM}$ MES-His, $0.01 \% \mathrm{HPMC}, 0.25 \mathrm{mM} \mathrm{KCl}$, and the integrated ISTDs: $7.5 \mathrm{mM}$ of $\mathrm{CsCl}$ and $\mathrm{LiCl}$. The sample consisted of $100 \mathrm{mM}$ MES-His, $0.01 \%$ HPMC, and one of six concentrations of $\mathrm{NaCl}(6,10,20,40,80$, and $140 \mathrm{mM})$. As the $\mathrm{NaCl}$ sample concentration increases, the magnitude of the "Cs" peak also increases, becoming asymmetrical and broad like the Na peak. Its mobility, however, remains unchanged. The magnitude of the "Li" peak continues to decrease in size (moving toward the baseline) until $\sim 40 \mathrm{mM}$ where the peak becomes inverted. Its mobility also continues to decrease as the width of the Na peak increases in size.

The linear theory of electromigration (LTEM) [21] reveals that system peaks are made up of linear combinations of the concentrations of each component in the sample; more precisely, of the differences between their concentrations in the BGE and the sample. This explains the observed variation in magnitudes of the "Cs" and "Li" integrated ISTD peaks when the amount of $\mathrm{NaCl}$ in the sample is varied. On the other hand, the arrival times of the system peaks should be independent of the sample composition according to the LTEM. While this is satisfied for the "Cs" peak, we speculate the shift in the migration time of the "Li" peak is due to a long comigration of the "Li" system zone with the Na sample zone. The comigration of zones is not handled by the LTEM, which 
assumes immediate separation of all zones upon the voltage application. This illustrates that both the nature and amount of integrated ISTD must be carefully chosen and tested across the sample's expected composition variations, to ensure that it remains relatively stable with altering the sample composition: specifically, (a) it does not become so large that it disrupts any analyte peaks; and (b) the integrated ISTD peak does not invert, making the peak area ratio ineffective.

\subsection{Designing the new BGE composition with Simul 5}

Mocked blood sample was defined as a solution of $140 \mathrm{mM}$ $\mathrm{NaCl}, 2.5 \mathrm{mM} \mathrm{CaCl}_{2}$, and $1.5 \mathrm{mM} \mathrm{MgCl}$, with added different concentrations of $\mathrm{KCl}$. While the large amount of $\mathrm{Na}+$ ions present in the mock blood sample keeps its composition relatively constant, it also results in a large asymmetrical peak that is broadened by electrodispersion. This makes the baseline more difficult to estimate-particularly if the analyte is in close proximity to the Na peak-and can lead to the dispersion of other peaks in the electropherogram. In an attempt to reduce the magnitude of this peak, one may consider adding a relatively small amount of $\mathrm{NaCl}$ to the $\mathrm{BGE}$, so that the difference in $\mathrm{Na}+$ ion concentration between the sample and BGE is reduced. We used Simul 5 to investigate this effect. The samples simulated in Figure S1 of the Supporting Information all contained $140 \mathrm{mM} \mathrm{NaCl}, 2.5 \mathrm{mM} \mathrm{CaCl}_{2}$, and $1.5 \mathrm{mM}$ $\mathrm{MgCl}_{2}$. The BGE was composed of $100 \mathrm{mM}$ MES-His, $2 \mathrm{mM}$ $\mathrm{CsCl}$, and either 0,10 , or $20 \mathrm{mM}$ of $\mathrm{NaCl}$.

The addition of $\mathrm{NaCl}$ to the BGE has a presumably unexpected effect on the $\mathrm{Na}$ analyte peak: rather than reducing its size, it reduces its effective mobility. The magnitude of the peak actually increases. Addition of a sample component ( $\mathrm{NaCl}$ in our case) into the BGE effectively turns the analyte peak into a system peak. According to the LTEM, the "Na" peak is close to the original position of the $\mathrm{Na}$ analyte peak at low $\mathrm{Na}$ concentrations in the BGE and moves away from this position as the Na concentration in the BGE increases. Again, the magnitude of the "Na" peak is governed by all constituents in both the sample and the BGE, so its magnitude may even increase when adding the Na sample component into the BGE. However, the "Na" peak appears later, which results in better peak shapes for the analytes that migrate faster than the $\mathrm{Na}$ zone. This is due to these analyte zones escaping the "Na" zone earlier, and thus are less influenced by electrodispersive effects.

Next, the mobilities of Cs (analyte), "Cs" (integrated ISTD), and K (analyte) peaks were investigated. Figure S2 of the Supporting Information plots three simulated electropherograms with either $5 \mathrm{mM} \mathrm{KCl}$ in the sample, $2 \mathrm{mM} \mathrm{CsCl}$ in the BGE (integrated ISTD), or $5 \mathrm{mM} \mathrm{CsCl}$ in the sample to visualize the overlap of these peaks if they were present in the same electropherogram. As expected from the previous work and Eq. (40) of Štědrý [22], the "Cs" integrated ISTD peak has a slightly slower effective mobility than its analyte peak. As the $\mathrm{K}$ peak is of interest, its effective mobility needed to

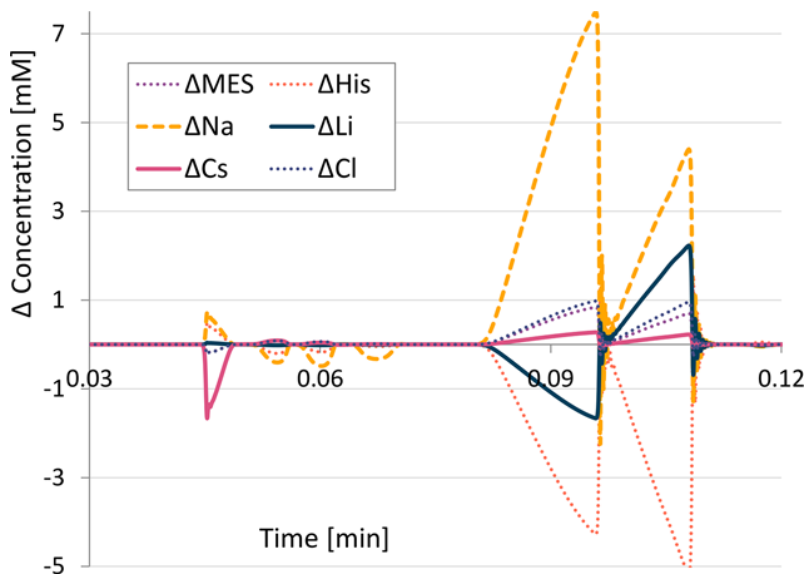

Figure 3. Simulation that demonstrates the effect of adding $2 \mathrm{mM}$ $\mathrm{Li}$ as an integrated ISTD when $20 \mathrm{mM} \mathrm{Na}$ is also present in the BGE. Addition of $\mathrm{Li}$ is seen to split the $\mathrm{Na}$ peak, and it becomes unclear which peak is the analyte peak and that is the Li integrated ISTD when looking at the compositions of each peak. In contrast, the Cs integrated ISTD peak is clearly the most depleted region of Cs ions. Thus, $\mathrm{Li}$ as an integrated ISTD was not investigated further and only Cs was used.

be reduced to resolve it from the "Cs" integrated ISTD peak. One common way of modifying the $\mathrm{K}+$ ion's mobility is by adding 18-crown-6 [23]. Ether additives cannot be modeled easily using Simul 5, however; so, the peak's mobility was manually adjusted from 76.2 to $68.2\left(10^{-9} \mathrm{~m}^{2} / \mathrm{V} \bullet \mathrm{s}\right)$ for the remaining simulations.

When used as an integrated ISTD previously, the "Li" peak performed nearly as well as "Cs"; thus, adding $\mathrm{LiCl}$ to the BGE was also investigated using Simul 5. If $20 \mathrm{mM}$ $\mathrm{NaCl}$ is also present in the BGE, then even small amounts of $\mathrm{LiCl}$ causes a very large, asymmetrical "Li" peak to appear (Figure S3 of Supporting Information). As the LiCl concentration in the BGE increases, the distance between the two system peaks also increases: the mobility of the first becoming faster while the second slows.

Figure 3 shows the change in the BGE constituents' concentrations (simulated) when $2 \mathrm{mM}$ of $\mathrm{LiCl}$ is added to the BGE. The "Cs" integrated ISTD peak is still clearly visible as the most depleted zone of Cs+ ions; however, it is not clear which of the subsequent peaks is the Na sample zone and that is the most depleted/accumulated zone of $\mathrm{Li}+$ ions. Thus, the "Li" peak cannot be used as an integrated ISTD in this work because the generated system peak is no longer deemed useful.

The final BGE used for modeling in Simul 5 was $100 \mathrm{mM}$ MES-His, $20 \mathrm{mM} \mathrm{NaCl}$, and $2 \mathrm{mM} \mathrm{CsCl}$. However, these concentrations were not optimized for the best separation; rather, they were values used to illustrate how the electropherograms would conceptually change with certain BGEadded constituents. When we moved away from simulations to practical use, the concepts developed using Simul 5 were applied to a few short experiments to find a BGE composition resulting in workable K analyte and "Cs" integrated ISTD 
peak shapes (see Supporting Information Fig. S4). This was found to be a BGE of $120 \mathrm{mM}$ MES-His, 0.01\% HPMC, $5 \mathrm{mM}$ $\mathrm{NaCl}, 2.5 \mathrm{mM}$ 18-crown-6, and $1 \mathrm{mM} \mathrm{CsCl}$. It should thereby be noted that this BGE has not been optimized for the best repeatability or performance. The motivation of this work was to demonstrate improvement in chip-to-chip RSDs using the integrated ISTD method, not to report best-case RSD values.

\subsection{Reproducibility of migration time}

Since all electropherograms were analyzed automatically with MATLAB algorithms, the migration times of the $\mathrm{K}$ analyte peak and "Cs" integrated ISTD peak were taken at three points out of curiosity: the start of the peak, at half the peak's height on the leading edge, and at the peak's maximum. Intra-chip RSDs for the arrival time of the $\mathrm{K}$ analyte peak were between $1.34 \%$ to $3.02 \%$ for each microchip (6 concentrations x 6 sample loads x 5 measurements per sample), with negligible difference between the determination methods. The chip-to-chip migration time RSD was 7.03\%, which is more than double the value for the analyte peak $(\mathrm{Na})$ in previous work [16]. The same holds true for the "Cs" integrated ISTD peak, which had a chip-to-chip migration time RSD of $2.34 \%$ in previous work, but $6.05 \%$ here. Since both peaks are similarly affected, this suggests either: (a) the BGE and electrophoretic protocols require further optimization; (b) this set of microchips are more variable than the set used previously; or (c) a combination of both.

Despite the increased variability in the individual peaks' migration times, the integrated ISTD improves the chip-tochip RSD from $7.03 \%$ to $0.89 \%$ when the ratio of the peak maximum times is used. With the ratio of times at halfmaximum, the chip-to-chip RSD is a bit more variable at $1.05 \%$; this further increases to $1.44 \%$ if the starting times of the peaks are used for the ratio. Since all electrophoretic data is computed automatically via script (rather than using manual integration), the starting time ratio may be more variable due to differences in the signal's derivative and/or noise that work opposite to each other within the same electropherogram. Using the migration time ratio at the peaks' maximums or half-maximums (on the leading edge), the chip-tochip RSD is comparable to the values found in previous work, which had better developed BGE and electrophoretic protocols, and a simpler sample solution. This further validates the integrated ISTD method as a good correction factor for improving the precision of analyte migration times.

Individual chip and chip-to-chip RSDs are provided in Table S1 of the Supporting Information.

\subsection{Linearity of peak areas and heights}

An ideal ISTD is a substance that is different from the analyte of interest, but which is influenced by other experimental conditions and sample preparation conditions in the same way. As mentioned above, we saw in previous work that the magnitude of the integrated ISTD peaks depended on the injected amount of sample (as desired); however, the peaks' magnitudes were also dependent on the sample composition and conductivity [16]. This resulted in a quadratic calibration curve for the peak area ratio versus analyte concentration, when a linear relationship is most-often desired for a constant sensitivity over the analyte quantification range. As described above, we expected in the present investigation that the large amount of $\mathrm{Na}+$ ions in the sample (relative to the other constituents) would result in a reference standard relationship closer to the desired linear relationship. To initially validate this, Simul 5 was used to model $\mathrm{KCl}$ variations between $2.5 \mathrm{mM}$ and $7.5 \mathrm{mM}$ in a sample containing $2.5 \mathrm{mM}$ $\mathrm{CaCl}_{2}, 1.5 \mathrm{mM} \mathrm{MgCl}_{2}$, and $140 \mathrm{mM} \mathrm{NaCl}$. The modeled BGE was as previous: $100 \mathrm{mM}$ MES-His, $20 \mathrm{mM} \mathrm{NaCl}$, and $2 \mathrm{mM}$ $\mathrm{CsCl}$. As expected, the varied $\mathrm{KCl}$ concentration had minimal change on the sample matrix properties: as the $\mathrm{K}$ peak increases, the other electropherogram peaks all remain primarily unchanged (Supporting Information Fig. S5). This is as one would hope for an ideal standard.

After collecting the experimental dataset, linear regressions were performed to build calibration curves for the six measured concentrations of $\mathrm{KCl}$. Correlation coefficients $\left(R^{2}\right)$ for each individual chip varied between 0.978 and 0.991 when using peak heights, and between 0.955 to 0.989 when using peak areas. These values are below the standard minimum of 0.99 for analytical method development due to variation in the peak area repeatability, as addressed in the next section. However, this study is aimed at developing a novel solution to improve chip-to-chip reproducibility in disposable devices, and not on method development of a specific analyte; thus, we are more interested in the relative improvement than the absolute values. Figure $4 \mathrm{~A}$ and $\mathrm{C}$ illustrate that the calibration lines vary strongly between chips, with differing slopes and offsets. At $8 \mathrm{mM}$, the difference in average $\mathrm{K}$ peak magnitude differs up to a factor of $1.8 \mathrm{x}$ or $1.5 \mathrm{x}$ when using heights or areas, respectively. This variation between chips is reflected in the chip-to-chip correlation coefficient for all measurements: 0.715 when using peak heights and 0.851 when using peak areas. These uncorrected chip-to-chip analyte values are very poor compared to previous work [16] on $\mathrm{NaCl}$ determination (0.970 for uncorrected $\mathrm{Na}$ analyte heights), which however forms no impediment to demonstrate the power of the integrated ISTD.

Using the height and area ratios of the $\mathrm{K}$ peak with the "Cs" integrated ISTD peak results in little-to-no improvement in each individual chip's correlation coefficient (Table 1). It is only when all data is included (hence chip-to-chip variations considered), that the ratios improve the linear fit. Individual chip coefficients are not improved because the peak ratios seem to offer little correction for run-to-run variation in peak sizes within a singular chip. The chip-to-chip correlation coefficients improve because the relationship between the $\mathrm{K}$ peak and "Cs" integrated ISTD peak seems to offer correction in the differing slopes/offsets, bringing the calibration curve for each chip closer together as illustrated in Figs. 4B and D. Correlation coefficients for the peak height ratio and 

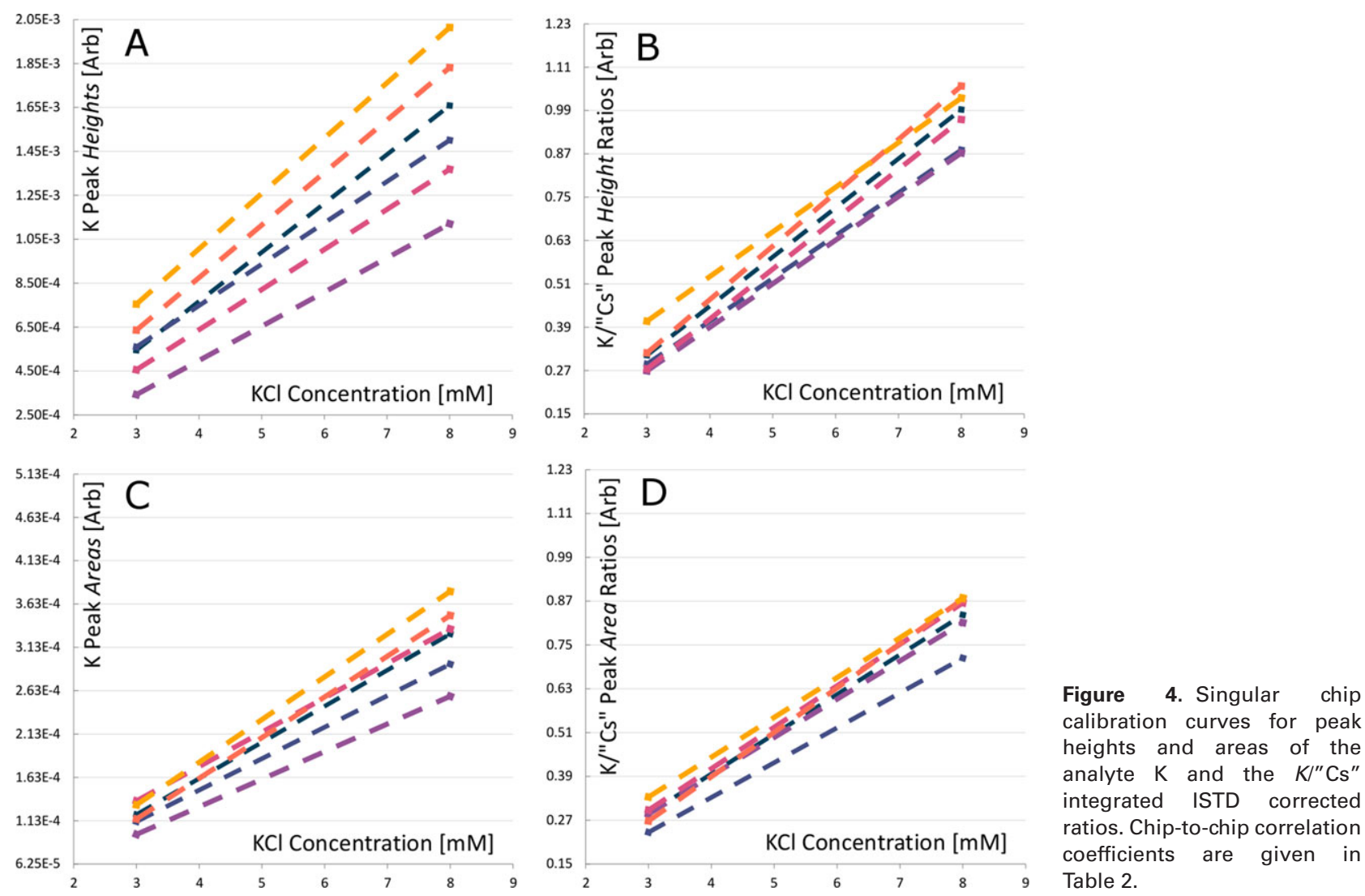

Table 1. Summary of linear regression fitting for the $\mathrm{KCl}$ quantification calibration curves

\begin{tabular}{|c|c|c|c|c|}
\hline & \multicolumn{2}{|c|}{ Peak area } & \multicolumn{2}{|c|}{ Peak height } \\
\hline & $R_{\mathrm{K}}^{2}$ & $R_{\mathrm{K} / " \mathrm{Cs} "}$ & $R_{\mathrm{K}}^{2}$ & $R^{2} \mathrm{~K} / \mathrm{Cs}^{\prime \prime}$ \\
\hline Chip 3-2 & 0.970 & 0.991 & 0.990 & 0.990 \\
\hline Chip 3-9* & 0.962 & 0.975 & 0.987 & 0.986 \\
\hline Chip 4-6 & 0.955 & 0.974 & 0.981 & 0.982 \\
\hline Chip 4-8 & 0.967 & 0.956 & 0.978 & 0.962 \\
\hline Chip 4-9 & 0.982 & 0.982 & 0.987 & 0.985 \\
\hline Chip 4-7 & 0.989 & 0.988 & 0.991 & 0.992 \\
\hline All Chips $(n=5)$ & 0.851 & $0.930(0.967)$ & 0.715 & 0.926 \\
\hline
\end{tabular}

The outlying chip (see Fig. 4D) is denoted with an asterisk and is removed for the peak area coefficient given in brackets. Chip naming refers to batch number and wafer position, illustrating no correlation in error due to fabrication.

peak area ratio are comparable: 0.926 and 0.930 , respectively. When inspecting the peak area ratio calibration curves for each microfluidic chip separately, one chip is empirically different from the rest (Fig. 4D). Using Dixon's Q test, chip 3-9 was identified as an outlier with a $90 \%$ confidence at 7 and $8 \mathrm{mM}$ of $\mathrm{KCl}$. Since $6 \mathrm{mM}$ is approximately the limit of quantification (peaks are $\sim 10 \mathrm{x}$ the signal's noise), it becomes difficult to mathematically demonstrate the chip as an outlier below this concentration level, despite being able to empirically see it from Fig. 4D. If chip 3-9's data is removed from the experiment set $(n[$ chips $]=5)$, then the correlation coefficient for the peak area ratio calibration curve improves from 0.930 to 0.966 . This is much improved from the uncorrected chip-to-chip peak area coefficient of 0.851 .

In previous work, one chip was also found to be an outlier. Unfortunately, since the microchip set was almost entirely different (the platinum conductivity electrodes were damaged between experiment sets), it is not possible to determine if the outlier is method and/or analyte dependent. However, it should be noted that the microfluidic chips fabricated for these papers were processed in an academic research setting, and yield was already low due to breakage, poor glass bonding, and defects. The prevalence of outliers may simply be due to the lack of industry-level process control in cleanroom fabrication.

\subsection{Repeatability of peak areas and heights}

The six microfluidic chips were loaded six times with six different $\mathrm{KCl}$ sample concentrations (3, 4, 5, 6, 7, $8 \mathrm{mM}$ ) to determine chip-to-chip peak height and area RSDs based on the Organisation for Economic Co-operation and Development requirements on analytical method validations [24]. Intrachip K peak heights (not shown) were the least variable with a median of approximately $3.5 \%$ deviation. When the analyte peak is small, it is known that peak height can be a more precise measure than peak area, due to difficulties in 

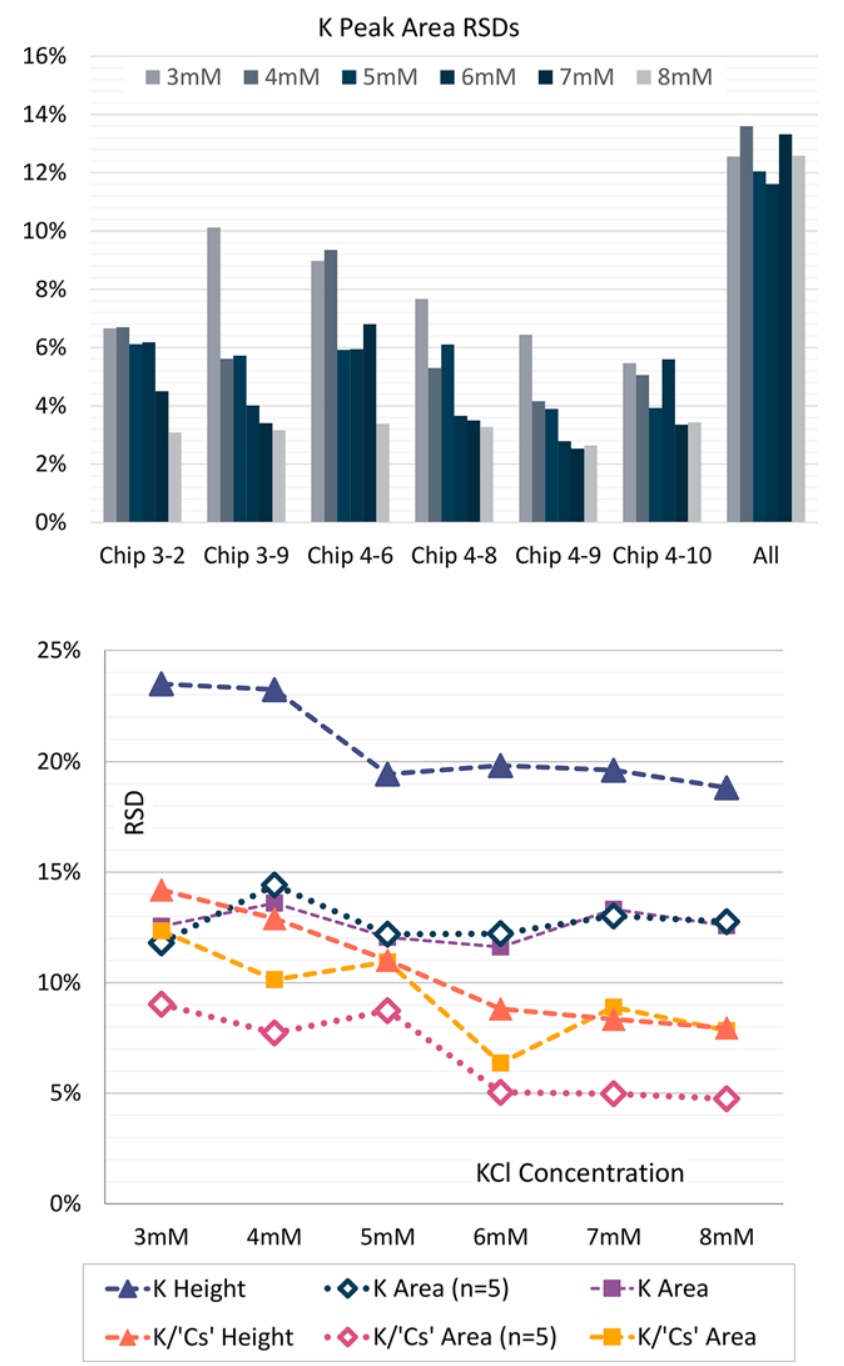

Figure 5. (Top) Comparison of the RSD in $K$ peak area at each $\mathrm{KCl}$ concentration on each individual microfluidic chip (30 measurements), to the peak area RSDs for all chips (180 measurements). Variation in peak areas within a singular chip has a median of $\sim 5.2 \%$, while the deviation in peak areas across all chips is above $\sim 12 \%$. This is as expected due to chip-to-chip differences. (Below) Illustration of how the RSDs improve at each $\mathrm{KCl}$ concentration if the "Cs" integrated ISTD is used to correct the $K$ peak measurement. Triangular points denote the improvement in peak height RSDs, square points in peak area RSDs, and diamond points in peak area RSDs if the outlier chip in Fig. 4D is removed $(n=5)$. Using the "Cs" integrated ISTD peak areas to correct the $K$ peak areas results in the best RSD values.

accurately defining the peak start/end points from the signal's noise [25]. However, this is only true when considering the same device. Chip-to-chip K peak height variation was 4$5 \mathrm{x}$ larger at each concentration than intrachip variation. In comparison, intra-chip K peak areas have a median of approximately 5.2\% deviation across multiple microchips (higher than intrachip K peak heights), but chip-to-chip peak areas are more precise than peak heights. This is expected: a short, broad peak and tall, thinner peak can have similar areas but much different heights. Figure 5 (top) compares intrachip
Table 2. Summary of chip-to-chip peak area and height RSDs for all experiments

\begin{tabular}{|c|c|c|c|c|c|}
\hline & \multicolumn{3}{|c|}{ Peak area RSDs } & \multicolumn{2}{|c|}{ Peak height RSDs } \\
\hline & $K$ & K/"Cs" & $K / " \mathrm{Cs} "(n=5)$ & $K$ & $K / " \mathrm{Cs} "$ \\
\hline $3 \mathrm{mM}$ & $12.6 \%$ & $12.35 \%$ & $9.03 \%$ & $23.5 \%$ & $14.2 \%$ \\
\hline $4 \mathrm{mM}$ & $13.6 \%$ & $10.1 \%$ & $7.72 \%$ & $23.2 \%$ & $12.9 \%$ \\
\hline $5 \mathrm{mM}$ & $12.1 \%$ & $8.21 \%$ & $8.74 \%$ & $19.4 \%$ & $11.0 \%$ \\
\hline $6 \mathrm{mM}$ & $11.6 \%$ & $6.37 \%$ & $5.04 \%$ & $19.8 \%$ & $8.82 \%$ \\
\hline $7 \mathrm{mM}$ & $13.3 \%$ & $8.90 \%$ & $4.98 \%$ & $19.6 \%$ & $8.35 \%$ \\
\hline $8 \mathrm{mM}$ & $12.6 \%$ & $7.83 \%$ & $4.75 \%$ & $18.8 \%$ & $7.95 \%$ \\
\hline$\rho$ & - & 0.655 & 0.831 & - & 0.891 \\
\hline
\end{tabular}

The outlying chip (see Fig. 4) is removed for the $K /{ }^{\prime \prime} \mathrm{Cs}$ " column of Peak Area RSDs denoted with $(n=5)$.

peak area RSDs to chip-to-chip peak area RSDs, illustrating that the variation when considering multiple disposable devices is much more signification than when only considering one microfluidic chip.

Next, integrated ISTD corrected RSDs are compared to the uncorrected analyte values. Comparing only SDs is not feasible due to scaling problems and statistical tests of significance for comparing RSDs are not readily available. However, for the purposes of this study, the visual data mining method suffices. In Fig. 5 (bottom), it can be seen that both peak height ratios and peak area ratios with the "Cs" integrated ISTD improve the chip-to-chip RSDs. Variations in K peak heights are reduced from $\sim 20 \%$ to $\sim 8 \%$ at higher concentrations $(6-8 \mathrm{mM})$ and $\sim 23 \%$ to $11 \sim 14 \%$ at lower $\mathrm{KCl}$ concentrations (3-5 mM). Similarly, variations in $\mathrm{K}$ peak areas are reduced from $12 \sim 14 \%$ (at all $\mathrm{KCl}$ concentrations) to $\sim 5 \%$ at higher concentrations $(6-8 \mathrm{mM})$ and to $8 \sim 9 \%$ at lower concentrations $(3-5 \mathrm{mM})$ when the outlier chip is excluded ( $n$ [chips] $=5)$. At $6 \mathrm{mM}$, the $\mathrm{K}$ peak's height is approximately 10x the signal's noise-one simple definition of LOQ [26]. Taking this into consideration, it is not unexpected that the imprecision of the measurement rises at $\mathrm{KCl}$ concentrations below this. Exact RSD values are given in Table 2 .

In previous work [15], we defined an experimental correlation coefficient $(\rho)$ based on simple error propagation to quantify how well an investigated ISTD improved the analyte's precision:

$\rho_{A \cdot I S T D}=\frac{R S D_{A}^{2}+R S D_{I S T D^{2}}-R S D_{P A R}^{2}}{2 R S D_{A} R S D_{I S T D}}$

where A is the analyte peak, ISTD is the traditional or integrated ISTD, and PAR is their ratio. Experimental correlation coefficients are calculated for each $\mathrm{KCl}$ concentration individually and then averaged, whereby $\rho=1$ would indicate perfect correlation and $\rho=0$ absolutely no correlation (both infeasible in practice). The peak height ratio coefficients range between 0.837 and 0.938 , averaging out to 0.891 . Without the outlier chip, the peak area ratio coefficients are between 0.654 and 0.935 , averaging out to 0.831 . These mean values are comparable to the $\mathrm{Na}$. $\mathrm{Cs}$ " and $\mathrm{Na}$. "Li" correlation coefficients (where $\mathrm{Na}$ was the analyte peak) found using the 
integrated ISTD method previously, further validating it as means for correcting imprecision in disposable microfluidic CE chips.

\section{Concluding remarks}

The integrated ISTD method has been further validated as a useful tool in correcting for chip-to-chip imprecision in pointof-care devices. When extended to samples with more constant compositions (here, mock human blood), the method is demonstrated to reliably improve the qualification (migration time precision) and quantification (size determination) of $\mathrm{K}$ with good linearity of both peak areas and heights. In samples with relatively high concentration changes of its components instead (e.g. human urine), special care will need to be taken to investigate the integrated ISTD's behavior over the sample's entire range: as shown in Fig. 2, the peak may invert. This can be determined quickly via simulation tools such as PeakMaster [27] before being verified experimentally. If the peak inversion cannot be prevented by adjusting the integrated ISTD's concentration in the BGE, then the addition of ISTD to the BGE will no longer be useful when used purely as a traditional ISTD replacement. However, this interesting side-effect may have other uses: for example, to identify operator errors (wrong cartridge, contamination of sample, etc.); or, to use one integrated ISTD for high sample conductivities and another for low. Adding a small amount of an overabundant sample ion to the BGE was also found to delay the migration time of the broad peak, leading to less dispersion in peaks that migrate before it. Further investigation needs to be done to see if this negatively impacts quantification of the overabundant ion.

It must be acknowledged that the microchip system presented here as a whole certainly cannot be immediately applied to point-of-care settings and the absolute precision values found are not yet useful for clinical requirements. In this study, benchtop electronics and a very basic microfluidic design were used over a design ready for point-of-care applications to limit unknown factors. Furthermore, time was also not spent investigating optimal electrophoretic protocols and background electrolyte design as these will vary with the microchip design and electronics. The goal was instead to further investigate a method for improving performance in microchip CE systems with a disposable "cartridge" design, without further complicating the microfluidic design or introducing additional error sources. Here, we showed that the integrated ISTD method meets these requirements.

This work was financially supported by a Spinoza grant (AvdB), the Czech Science Foundation grant no. 15-18424Y and CEEPUS Network no.: CIII-RO-0010-13-1819.

The authors have declared no conflict of interest.

\section{References}

[1] Ryvolová, M., Macka, M., Brabazon, D., Preisler, J., Trends Anal. Chem. 2010, 29, 339-353.

[2] Breadmore, M. C., J. Chromatogr. A 2012, 1221, 4255.

[3] Kubáň, P., Hauser, P. C., Electrophoresis 2011, 32, 30 42.

[4] Eijkel, J. C. T., Bioanalysis 2015, 7, 1385-1387.

[5] Vrouwe, E. X., Luttge, R., Olthuis, W., van den Berg, A., Electrophoresis 2005, 26, 3032-3042.

[6] Floris, A., Staal, S., Lenk, S., Staijen, E., Kohlheyer, D., Eijkel, J. C. T., van den Berg, A., Lab Chip 2010, 10, 1799-1806.

[7] Muñoz, M. Á., Floris, J., Staal, S. S., Ríos, Á., Eijkel, J. C. T., van den Berg, A., Electrophoresis 2013, 34, 29562961.

[8] Staal, S., Ungerer, M., Floris, A., Ten Brinke, H.-W., Helmhout, R., Tellegen, M., Janssen, K., Karstens, E., van Arragon, C., Lenk, S., Staijen, E., Bartholomew, J., Krabbe, H., Movig, K., Dubský, P., van den Berg, A., Eijkel, J., Electrophoresis 2015, 36, 712 721.

[9] Ren, K., Zhou, J., Wu, H., Acc. Chem. Res. 2013, 46, 2396-2406.

[10] Altria, K. D., Glaxosmithkline, R., LC-GC Eur. 2002, 15, 588-594.

[11] Dose, E. V., Guiochon, G. A., Anal. Chem. 1991, 63, 1154-1158.

[12] Blanco-Heras, G. A., Turnes-Carou, M. I., LópezMahía, P., Muniategui-Lorenzo, S., Prada-Rodríguez, D., Fernández-Fernández, E., Electrophoresis 2008, 29, 1347-1354.

[13] Revermann, T., Götz, S., Künnemeyer, J., Karst, U., Analyst 2008, 133, 167-174.

[14] Masár, M., Bomastyk, B., Bodor, R., Horčičiak, M., Danč, L., Troška, P., Kuss, H.-M., Microchim. Acta 2012, 177, 309-316.

[15] Bidulock, A. C. E., van den Berg, A., Eijkel, J. C. T., Electrophoresis 2015, 36, 875-883.

[16] Bidulock, A. C. E., Dubský, P., Van Den Berg, A., Eijkel, J. C. T., Anal. Chem. 2017, 89, 2886-2892.

[17] Gaš, B., Hruška, V., Dittmann, M., Bek, F., Witt, K., J. Sep. Sci. 2007, 30, 1435-1445.

[18] Vrouwe, E. X., Quantitative Microchip Capillary Electrophoresis for Inorganic Ion Analysis at the Point of Care, Febodruk BV, Enschede, Netherlands 2005.

[19] Mazet, V., Carteret, C., Brie, D., Idier, J., Humbert, B., Chemom. Intell. Lab. Syst. 2005, 76, 121-133.

[20] Hruška, V., Jaroš, M., Gaš, B., Electrophoresis 2006, 27, 984-991.

[21] Štědrý, M., Jaroš, M., Hruška, V., Gaš, B., Electrophoresis 2004, 25, 3071-3079.

[22] Štědrý, M., Jaroš, M., Včeláková, K., Gaš, B., Electrophoresis 2003, 24, 536-547.

[23] Francois, C., Morin, P., Dreux, M., J. Chromatogr. A 1995, 706, 535-553. 
[24] Guidance document for single laboratory validation of quantitative analytical methods - guidance used in support of pre-and-post-registration data requirements for plant protection and biocidal products, ENV/JM/MONO, 2014, 20, 23-25.

[25] Wätzig, H., J. Chromatogr. A 1995, 700, 1-7.
[26] Carlson, J., Wysoczanski, A., Voigtman, E., Spectrochim. Acta Part B 2014, 96, 69-73.

[27] The Group of Electrophoretic and Chromatographic Separation Methods: Software Downloads, can be found at https://echmet.natur.cuni.cz/software/download, 2018. 